\title{
AN EXTENSION AND A REFINEMENT OF VAN DER CORPUT'S INEQUALITY
}

\author{
JIAN CAO, DA-WEI NIU, AND FENG QI
}

Received 1 April 2006; Revised 18 June 2006; Accepted 22 June 2006

van der Corput's inequality is extended and refined by using Euler-Maclaurin formula and other analytic techniques.

Copyright (c) 2006 Hindawi Publishing Corporation. All rights reserved.

\section{Introduction}

Let $S_{n}=\sum_{k=1}^{n}(1 / k)$ and $a_{n} \geq 0$ for $n \in \mathbb{N}$ such that $0<\sum_{n=1}^{\infty} a_{n}<\infty$. The famous van der Corput inequality [10] reads that

$$
\sum_{n=1}^{\infty}\left(\prod_{k=1}^{n} a_{k}^{1 / k}\right)^{1 / S_{n}}<e^{1+\gamma} \sum_{n=1}^{\infty}(n+1) a_{n},
$$

where $\gamma=0.57721566 \ldots$ stands for Euler-Mascheroni constant. The constant $e^{1+\gamma}$ in (1.1) is the best possible.

$\mathrm{Hu}[5]$ gave a strengthened version of (1.1) as

$$
\sum_{n=1}^{\infty}\left(\prod_{k=1}^{n} a_{k}^{1 / k}\right)^{1 / S_{n}}<e^{1+\gamma} \sum_{n=1}^{\infty}\left(n-\frac{\ln n}{4}\right) a_{n} .
$$

Yang [14] established a relation between Carleman's inequality and van der Corput's inequality and presented the following:

$$
\sum_{n=1}^{\infty}\left(\prod_{k=1}^{n} a_{k}^{1 / k^{\alpha}}\right)^{1 / S_{n}(\alpha)}<e \sum_{n=1}^{\infty} e^{\alpha n^{\alpha-1} S_{n}(\alpha)} a_{n},
$$

where $S_{n}(\alpha)=\sum_{k=1}^{n}\left(1 / k^{\alpha}\right)$ and $\alpha \in[0,1]$. 
2 An extension and a refinement of van der Corput's inequality

In a recent paper [15], Yang has obtained another extension of (1.1) as follows:

$$
\sum_{n=1}^{\infty}\left(\prod_{k=1}^{n} a_{k}^{1 /(k+\beta)}\right)^{1 / S_{n}(\beta)}<e^{1+\gamma_{1}(\beta)} \sum_{n=1}^{\infty}\left(n+\frac{1}{2}+\beta\right) a_{n},
$$

where $\beta \in(-1, \infty), S_{n}(\beta)=\sum_{k=1}^{n}(1 /(k+\beta))$, and

$$
\gamma_{1}(\beta)=\lim _{n \rightarrow \infty}\left[\sum_{k=1}^{n} \frac{1}{k+\beta}-\ln (n+\beta)\right] .
$$

Applying $\beta=0$ in (1.4) leads to

$$
\sum_{n=1}^{\infty}\left(\prod_{k=1}^{n} a_{k}^{1 / k}\right)^{1 / S_{n}}<e^{1+\gamma} \sum_{n=1}^{\infty}\left(n+\frac{1}{2}\right) a_{n}
$$

which improved inequality (1.1) clearly.

For more information about van der Corput's inequality, please refer to $[2,5,10,14$, $15]$ and the references therein.

The aim of this paper is to further extend and refine van der Corput's inequality by using Euler-Maclaurin formula and other analytic techniques.

Our main results are the following two theorems.

Theorem 1.1. Let $a_{n} \geq 0$ for $n \in \mathbb{N}$ such that $0 \leq \sum_{n=1}^{\infty} a_{n}<\infty$. Then

$$
\begin{aligned}
& \sum_{n=1}^{\infty}\left[\prod_{k=1}^{n} a_{k}^{1 / \sqrt{k(k+\lambda)}}\right]^{1 / S_{n}(\lambda)} \\
& \quad<e^{1+(1+\lambda / 3) \gamma(\lambda)} \sum_{n=1}^{\infty}(n+1)^{\lambda / 3}\left[1-\frac{\ln (n+1)}{4(n+1+\lambda / 2)}\right] a_{n},
\end{aligned}
$$

where $\lambda \in[0, \infty)$,

$$
\begin{gathered}
S_{n}(\lambda)=\sum_{k=1}^{n} \frac{1}{\sqrt{k(k+\lambda)}}, \\
\gamma(\lambda)=\lim _{n \rightarrow \infty}\left[S_{n}(\lambda)-2 \ln \frac{\sqrt{n}+\sqrt{n+\lambda}}{1+\sqrt{1+\lambda}}\right] .
\end{gathered}
$$

Theorem 1.2. Let $a_{n} \geq 0$ for $n \in \mathbb{N}$ such that $0 \leq \sum_{n=1}^{\infty} a_{n}<\infty$. Then

$$
\sum_{n=1}^{\infty}\left(\prod_{k=1}^{n} a_{k}^{1 / k}\right)^{1 / S_{n}}<e^{1+\gamma} \sum_{n=1}^{\infty} n\left(1-\frac{\ln n}{3 n-1 / 4}\right) a_{n} .
$$

Remark 1.3. It is easy to see that inequality (1.10) refines inequalities (1.1), (1.2), and (1.6). 


\section{Lemmas}

To prove our main results, the following lemmas are necessary.

Recall $[7,9]$ that a function $f$ is called completely monotonic on an interval $I$ if $f$ has derivatives of all orders on $I$ and $0<(-1)^{k} f^{(k)}(x)<\infty$ for all $k \geq 0$ on $I$. The background information and an extensive bibliography about the theory of completely monotonic function can be found in the recent papers $[4,7,8]$.

Lemma 2.1. The function $f(x)=1 / \sqrt{x(x+\lambda)}$ for $\lambda \in[0, \infty)$ is completely monotonic in $(0, \infty)$ and $\lim _{x \rightarrow \infty} f^{(i)}(x)=0$ for any nonnegative integer $i$.

Proof. It is not difficult to verify that the functions $1 / \sqrt{x}$ and $1 / \sqrt{x+\lambda}$ are completely monotonic in $x \in(0, \infty)$. Since the product of any finite completely monotonic functions is also strictly completely monotonic (see [11]), then the function $f(x)$ is strictly completely monotonic in $(0, \infty)$.

By induction, it is easy to verify that $\lim _{x \rightarrow \infty} f^{(i)}(x)=0$ holds for any nonnegative integer $i$. The proof of Lemma 2.1 is complete.

Recall that Euler-Maclaurin formula (see [1, pages 617-623] and $[6,12,14]$ ) states

$$
\sum_{k=1}^{n} f(k)=\int_{1}^{n} f(x) \mathrm{d} x+\frac{1}{2}[f(n)+f(1)]+\int_{1}^{n} \rho_{1}(x) f^{\prime}(x) \mathrm{d} x,
$$

where $\rho_{1}(x)=x-[x]+1 / 2$ is Bernoulli's function and $f \in C^{1}[1, \infty)$. Furthermore, if $(-1)^{i} f^{(i)}(x)>0$ and $\lim _{x \rightarrow \infty} f^{(i)}(x)=0$ for $i=1,2,3$, then

$$
\int_{n}^{\infty} \rho_{1}(x) f^{\prime}(x) \mathrm{d} x=-\frac{1}{12} f^{\prime}(n) \epsilon, \quad 0<\epsilon<1 .
$$

Lemma 2.2. For $n \in \mathbb{N}$ and $\lambda \in[0, \infty)$,

$$
S_{n}(\lambda)<\ln (n+1)+\gamma(\lambda),
$$

where $S_{n}(\lambda)$ and $\gamma(\lambda)$ are defined by (1.8) and (1.9), respectively.

Proof. It is clear that Lemma 2.1 allows us to apply Euler-Maclaurin formula (2.1) and formula (2.2) to $f(x)=1 / \sqrt{x(x+\lambda)}$. From this, it follows that

$$
\begin{gathered}
S_{n}(\lambda)=2 \ln \frac{\sqrt{n}+\sqrt{n+\lambda}}{1+\sqrt{1+\lambda}}+\frac{1}{2}\left[\frac{1}{\sqrt{1+\lambda}}+\frac{1}{\sqrt{n(n+\lambda)}}\right] \\
+\int_{1}^{n} \rho_{1}(x)\left[\frac{1}{\sqrt{x(x+\lambda)}}\right]^{\prime} \mathrm{d} x, \\
\int_{n}^{\infty} \rho_{1}(x)\left[\frac{1}{\sqrt{x(x+\lambda)}}\right]^{\prime} \mathrm{d} x=-\frac{1}{12}\left[\frac{1}{\sqrt{n(n+\lambda)}}\right]^{\prime} \epsilon=\frac{(2 n+\lambda) \epsilon}{24[n(n+\lambda)]^{3 / 2}},
\end{gathered}
$$

where $0<\epsilon<1$, and

$$
\gamma(\lambda)=\frac{1}{2 \sqrt{1+\lambda}}+\int_{1}^{\infty} \rho_{1}(x)\left[\frac{1}{\sqrt{x(x+\lambda)}}\right]^{\prime} \mathrm{d} x .
$$


4 An extension and a refinement of van der Corput's inequality

Therefore,

$$
\begin{aligned}
S_{n}(\lambda) & =2 \ln \frac{\sqrt{n}+\sqrt{n+\lambda}}{1+\sqrt{1+\lambda}}+\gamma(\lambda)-\frac{(2 n+\lambda) \epsilon}{24[n(n+\lambda)]^{3 / 2}}+\frac{1}{2 \sqrt{n(n+\lambda)}} \\
& <\ln n+\gamma(\lambda)+\frac{1}{2 \sqrt{n(n+\lambda)}},
\end{aligned}
$$

and then

$$
\begin{aligned}
S_{n}(\lambda) & =\sum_{k=1}^{n+1} \frac{1}{\sqrt{k(k+\lambda)}}-\frac{1}{\sqrt{(n+1)(n+1+\lambda)}} \\
& <\ln (n+1)+\gamma(\lambda)-\frac{1}{2 \sqrt{(n+1)(n+1+\lambda)}}<\ln (n+1)+\gamma(\lambda) .
\end{aligned}
$$

The proof of Lemma 2.2 is complete.

Lemma 2.3. For $k \in \mathbb{N}$ and $\lambda \in[0, \infty)$,

$$
\begin{gathered}
\sqrt{\frac{k(k+\lambda)}{(k+1)(k+1+\lambda)}} \leq \frac{k+\lambda / 2}{k+1+\lambda / 2}, \\
\sqrt{(k+1)(k+1+\lambda)}-\sqrt{k(k+\lambda)} \leq 1+\frac{\lambda}{3} .
\end{gathered}
$$

Proof. Inequality (2.8) is equivalent to

$$
\left(k+\frac{\lambda}{2}\right)^{2}(k+1)(k+1+\lambda) \geq k(k+\lambda)\left(k+1+\frac{\lambda}{2}\right)^{2} .
$$

The difference between both sides of (2.10) equals

$$
\begin{gathered}
{\left[k^{4}+2 k^{3}(\lambda+1)+k^{2}\left(\frac{5}{4} \lambda^{2}+3 \lambda+1\right)+k\left(\frac{\lambda^{3}}{4}+\frac{3}{2} \lambda^{2}+\lambda\right)+\frac{\lambda+1}{4} \lambda^{2}\right]} \\
-\left[k^{4}+2 k^{3}(\lambda+1)+k^{2}\left(\frac{5}{4} \lambda^{2}+3 \lambda+1\right)+k\left(\frac{\lambda^{3}}{4}+\lambda^{2}+\lambda\right)\right] \\
=\frac{k \lambda^{2}}{2}+\frac{\lambda^{2}}{4}+\frac{\lambda^{3}}{4} \geq 0 .
\end{gathered}
$$

Inequality (2.9) can be deduced straightforwardly from

$$
\begin{aligned}
& \sqrt{(k+1)(k+1+\lambda)}-\sqrt{k(k+\lambda)} \\
& \quad=\frac{2 k+\lambda+1}{\sqrt{(k+1)(k+1+\lambda)}+\sqrt{k(k+\lambda)}} \leq 1+\frac{\lambda}{3} .
\end{aligned}
$$

The proof of Lemma 2.3 is complete. 
Jian Cao et al. 5

Lemma 2.4. For $x \in(0, \infty)$ and $\lambda \in[0, \infty)$,

$$
\left[1-\frac{1}{2(x+1+\lambda / 2)}\right]^{\ln (x+1)}<1-\frac{\ln (x+1)}{4(x+1+\lambda / 2)} \text {. }
$$

Proof. Let $u(x, \lambda)=2(x+1+\lambda / 2)-\ln (x+1)$ for $x \in(0, \infty)$ and $\lambda \in[0, \infty)$. Then $\partial u(x, \lambda) / \partial x=2-1 /(x+1)>0$ and $u(0, \lambda)=2+\lambda>0$. Thus,

$$
\frac{\ln ^{2}(x+1)}{8(x+1+\lambda / 2)^{2}}<\frac{\ln (x+1)}{4(x+1+\lambda / 2)} .
$$

As a result, by

$$
\left(1-\frac{1}{t}\right)^{-t}>e
$$

for $t>1$ and

$$
e^{t}<1+t+\frac{t^{2}}{2}
$$

for $t<0$, it follows that

$$
\begin{aligned}
{\left[1-\frac{1}{2(x+1+\lambda / 2)}\right]^{\ln (x+1)} } & <\left(\frac{1}{e}\right)^{\ln (x+1) / 2(x+1+\lambda / 2)} \\
& <1-\frac{\ln (x+1)}{2(x+1+\lambda / 2)}+\frac{\ln ^{2}(x+1)}{8(x+1+\lambda / 2)^{2}}<1-\frac{\ln (x+1)}{4(x+1+\lambda / 2)} .
\end{aligned}
$$

The proof of Lemma 2.4 is complete.

Lemma 2.5. For $k \in \mathbb{N}$ and $\lambda \in[0, \infty)$,

$$
\begin{aligned}
B_{k}(\lambda) & \triangleq\left[\frac{\sqrt{(k+1)(k+1+\lambda)} S_{k+1}(\lambda)}{\sqrt{k(k+\lambda)} S_{k}(\lambda)}\right]^{\sqrt{k(k+\lambda)} S_{k}(\lambda)} \\
& \leq e^{1+(1+\lambda / 3) \gamma(\lambda)}(k+1)^{1+\lambda / 3}\left[1-\frac{\ln (k+1)}{4(k+1+\lambda / 2)}\right] .
\end{aligned}
$$

Proof. For $k \in \mathbb{N}$,

$$
B_{k}(\lambda)=\left\{1+\frac{1+[\sqrt{(k+1)(k+1+\lambda)}-\sqrt{k(k+\lambda)}] S_{k}(\lambda)}{\sqrt{k(k+\lambda)} S_{k}(\lambda)}\right\}^{\sqrt{k(k+\lambda)} S_{k}(\lambda)} \triangleq C_{k}^{h(k, \lambda)},
$$

where

$$
\begin{aligned}
& C_{k}=\left[1+\frac{1}{g(k, \lambda)}\right]^{g(k, \lambda)}, \quad g(k, \lambda)=\frac{\sqrt{k(k+\lambda)} S_{k}(\lambda)}{h(k, \lambda)}, \\
& h(k, \lambda)=1+[\sqrt{(k+1)(k+1+\lambda)}-\sqrt{k(k+\lambda)}] S_{k}(\lambda) .
\end{aligned}
$$


6 An extension and a refinement of van der Corput's inequality

It is easy to see that

$$
\begin{aligned}
g(k, \lambda)+1 & =\frac{1+\sqrt{(k+1)(k+1+\lambda)} S_{k}(\lambda)}{1+[\sqrt{(k+1)(k+1+\lambda)}-\sqrt{k(k+\lambda)}] S_{k}(\lambda)} \\
& \leq \frac{\sqrt{(k+1)(k+1+\lambda)}}{\sqrt{(k+1)(k+1+\lambda)}-\sqrt{k(k+\lambda)}} .
\end{aligned}
$$

By using the inequality $(1+1 / x)^{x}<e[1-1 / 2(x+1)]$ obtained in [13], inequalities $(2.21)$ and (2.8) in Lemma 2.3, it is deduced that

$$
\begin{aligned}
C_{k} & =\left[1+\frac{1}{g(k, \lambda)}\right]^{g(k, \lambda)} \leq e\left\{1-\frac{1}{2[g(k, \lambda)+1]}\right\} \\
& \leq e\left[\frac{1}{2}+\frac{\sqrt{k(k+\lambda)}}{2 \sqrt{(k+1)(k+1+\lambda)}}\right] \leq e\left[1-\frac{1}{2(k+1+\lambda / 2)}\right] .
\end{aligned}
$$

Hence, from inequalities (2.3), (2.9), (2.22) in Lemma 2.2, and (2.13) in Lemma 2.4, it is shown that

$$
\begin{aligned}
B_{k}(\lambda) & \leq\left\{e\left[1-\frac{1}{2(k+1+\lambda / 2)}\right]\right\}^{h(k, \lambda)} \\
& \leq\left\{e\left[1-\frac{1}{2(k+1+\lambda / 2)}\right]\right\}^{1+(1+\lambda / 3)[\ln (k+1)+\gamma(\lambda)]} \\
& \leq e^{1+(1+\lambda / 3) \gamma(\lambda)}(k+1)^{1+\lambda / 3}\left[1-\frac{1}{2(k+1+\lambda / 2)}\right] \\
& \leq e^{1+(1+\lambda / 3) \gamma(\lambda)}(k+1)^{1+\lambda / 3}\left[1-\frac{\ln (k+1)}{4(k+1+\lambda / 2)}\right] .
\end{aligned}
$$

The proof of Lemma 2.5 is complete.

Lemma 2.6. For $n \in \mathbb{N}$,

$$
\left(1-\frac{1}{2 n+11 / 6}\right)^{\ln n} \leq 1-\frac{\ln n}{3 n-1 / 4}
$$

Proof. For $n=1$, inequality (2.24) holds clearly. For $n=2$,

$$
1-\frac{\ln 2}{6-1 / 4}-\left(1-\frac{1}{4+11 / 6}\right)^{\ln 2}=0.0016626 \ldots>0
$$

inequality (2.24) holds also. 
For $n \geq 3$, by using (2.15) and (2.16), it is shown that

$$
\left(1-\frac{1}{2 n+11 / 6}\right)^{\ln n}<e^{-\ln n /(2 n+11 / 6)}<1-\frac{\ln n}{2 n+11 / 6}+\frac{\ln ^{2} n}{2(2 n+11 / 6)^{2}} .
$$

So, it is sufficient to prove that

$$
1-\frac{\ln n}{2 n+11 / 6}+\frac{\ln ^{2} n}{2(2 n+11 / 6)^{2}}<1-\frac{\ln n}{3 n-1 / 4}
$$

for $n \geq 3$. For this purpose, let $m=n-1 / 12$, then inequality (2.27) can be rearranged as

$$
g(m) \triangleq \frac{4 m}{3}-\frac{4}{3}-\frac{8}{3 m}-\ln \left(m+\frac{1}{12}\right)>0 .
$$

Differentiation of $g(x)$ gives

$$
g^{\prime}(x)=\frac{4}{3}+\frac{8}{3 x^{2}}-\frac{1}{x+1 / 12}>0
$$

This means that $g(m)$ is increasing. Further, since

$$
g\left(3-\frac{1}{12}\right)=\frac{4}{3}\left(3-\frac{1}{12}\right)-\frac{4}{3}-\frac{8}{3(3-1 / 12)}-\ln 3=0.5426575526 \ldots>0
$$

then $g(m)$ is positive for $m \geq 3$. The proof of Lemma 2.6 is complete.

\section{Proofs of theorems}

Proof of Theorem 1.1. Setting $c_{k}>0$ for $1 \leq k \leq n$ and letting

$$
\left[\prod_{k=1}^{n} c_{k}^{1 / \sqrt{k(k+\lambda)}}\right]^{-1 / S_{n}(\lambda)}=\frac{1}{\sqrt{(n+1)(n+1+\lambda)} S_{n+1}(\lambda)},
$$

then

$$
c_{k}=\frac{\left[\sqrt{(k+1)(k+1+\lambda)} S_{k+1}(\lambda)\right]^{\sqrt{k(k+\lambda)} S_{k}(\lambda)}}{\left[\sqrt{k(k+\lambda)} S_{k}(\lambda)\right]^{\sqrt{k(k+\lambda)} S_{k-1}(\lambda)}} .
$$


8 An extension and a refinement of van der Corput's inequality

Using the discrete weighted arithmetic-geometric mean inequality and (3.2) and interchanging the order of summation yield

$$
\begin{aligned}
\sum_{n=1}^{\infty}( & \left.\prod_{k=1}^{n} a_{k}^{1 / \sqrt{k(k+\lambda)}}\right)^{1 / S_{n}(\lambda)} \\
& =\sum_{n=1}^{\infty}\left(\prod_{k=1}^{n}\left(c_{k} a_{k}\right)^{1 / \sqrt{k(k+\lambda)}}\right)^{1 / S_{n}(\lambda)}\left(\prod_{k=1}^{n} c_{k}^{1 / \sqrt{k(k+\lambda)}}\right)^{-1 / S_{n}(\lambda)} \\
& \leq \sum_{n=1}^{\infty} \sum_{k=1}^{n} \frac{1}{\sqrt{k(k+\lambda)} S_{n}(\lambda)} c_{k} a_{k} \frac{1}{\sqrt{(n+1)(n+1+\lambda)} S_{n+1}(\lambda)} \\
& =\sum_{k=1}^{\infty} \frac{1}{\sqrt{k(k+\lambda)}} c_{k} a_{k} \sum_{n=k}^{\infty} \frac{1}{\sqrt{(n+1)(n+1+\lambda)} S_{n}(\lambda) S_{n+1}(\lambda)} \\
& =\sum_{k=1}^{\infty} \frac{1}{\sqrt{k(k+\lambda)}} c_{k} a_{k} \sum_{n=k}^{\infty}\left[\frac{1}{S_{n}(\lambda)}-\frac{1}{S_{n+1}(\lambda)}\right] \\
& =\sum_{k=1}^{\infty} \frac{1}{\sqrt{k(k+\lambda)}} c_{k} a_{k} \frac{1}{S_{k}(\lambda)} \\
& =\sum_{k=1}^{\infty}\left[\frac{\sqrt{(k+1)(k+1+\lambda)} S_{k+1}(\lambda)}{\sqrt{k(k+\lambda)} S_{k}(\lambda)}\right]^{\sqrt{k(k+\lambda)} S_{k}(\lambda)} a_{k} .
\end{aligned}
$$

Applying inequality (2.18) in the final line of (3.3) gives inequality (1.7). The proof of Theorem 1.1 is complete.

Proof of Theorem 1.2. It is easy to see that

$$
B_{1}(0)=3<e^{1+\gamma}, \quad B_{2}(0)=\left(\frac{11}{6}\right)^{3}<e^{1+\gamma} \cdot 2\left(1-\frac{\ln 2}{6-1 / 4}\right) .
$$

For $n \geq 3$, inequality

$$
e^{1 / 2 n}\left(1-\frac{1}{2 n+11 / 6}\right)^{1+\gamma}<e^{1 / 2 n} e^{-(1+\gamma) /(2 n+11 / 6)}<1
$$

follows from using an inequality

$$
\left(1+\frac{1}{x}\right)^{x}<e\left(1-\frac{1}{2 x+11 / 6}\right)
$$


in [3]. By (2.3), (3.6), Lemma 2.6, and inequality (3.5),

$$
\begin{aligned}
B_{n}(0) & =\left[\frac{(n+1) S_{n}+1}{n S_{n}}\right]^{n S_{n}}=\left\{\left[1+\frac{1}{n S_{n} /\left(S_{n}+1\right)}\right]^{n S_{n} /\left(S_{n}+1\right)}\right\}^{S_{n}+1} \\
& <\left\{e\left[1-\frac{1}{2 n S_{n} /\left(S_{n}+1\right)+11 / 6}\right]\right\}^{S_{n}+1} \\
& <\left\{e\left[1-\frac{1}{2 n S_{n} /\left(S_{n}+1\right)+11 / 6}\right]\right\}^{1+\ln n+\gamma+1 / 2 n} \\
& <\left[e\left(1-\frac{1}{2 n+11 / 6}\right)\right]^{1+\ln n+\gamma+1 / 2 n} \\
& <e^{1+\gamma} n e^{1 / 2 n}\left(1-\frac{1}{2 n+11 / 6}\right)^{1+\ln n+\gamma} \\
& <e^{1+\gamma} n\left(1-\frac{\ln n}{3 n-1 / 4}\right) .
\end{aligned}
$$

Taking $\lambda=0$ in inequality (3.3) yields

$$
\begin{aligned}
\sum_{n=1}^{\infty}\left(\prod_{k=1}^{n} a_{k}^{1 / k}\right)^{1 / S_{n}} & \leq \sum_{n=1}^{\infty}\left[\frac{(n+1) S_{n+1}}{n S_{n}}\right]^{n S_{n}} a_{n} \\
& =\sum_{n=1}^{\infty} B_{n}(0) a_{n}<e^{1+\gamma} \sum_{n=1}^{\infty} n\left(1-\frac{\ln n}{3 n-1 / 4}\right) a_{n} .
\end{aligned}
$$

The proof of Theorem 1.2 is complete.

\section{Acknowledgments}

The authors would like to heartily express many thanks to three anonymous referees for their many detailed comments on this paper and recommending some references to us. The authors were supported in part by the Science Foundation of the Project for Fostering Innovation Talents at Universities of Henan Province, China.

\section{References}

[1] G. E. Andrews, R. Askey, and R. Roy, Special Functions, Encyclopedia of Mathematics and Its Applications, vol. 71, Cambridge University Press, Cambridge, 1999.

[2] B. Autora, Publication list: J. G. van der Corput, Acta Arithmetica 36 (1980), no. 1, 91-99.

[3] Ch.-P. Chen and F. Qi, On further sharpening of Carleman's inequality, Dàxué Shùxué (College Mathematics) 21 (2005), no. 2, 88-90 (Chinese).

[4] A. Z. Grinshpan and M. E. H. Ismail, Completely monotonic functions involving the gamma and q-gamma functions, Proceedings of the American Mathematical Society 134 (2006), no. 4, 11531160.

[5] K. Hu, On the van der Corput inequality, Journal of Mathematics (Shùxué Zázhì) 23 (2003), no. 1, 126-128 (Chinese).

[6] J.-Ch. Kuang, Asymptoic estimations of finite sums, Journal of Hexi University 2 (2002), no. 2, 1-8 (Chinese). 


\section{An extension and a refinement of van der Corput's inequality}

[7] F. Qi, Certain logarithmically $N$-alternating monotonic functions involving gamma and q-gamma functions, RGMIA Research Report Collection 8 (2005), no. 3, article 5, available online at http://rgmia.vu.edu.au/v8n3.html.

[8] F. Qi and B.-N. Guo, Complete monotonicities of functions involving the gamma and digamma functions, RGMIA Research Report Collection 7 (2004), no. 1, article 8, 63-72, available online at http://rgmia.vu.edu.au/v7n3.html.

[9] F. Qi, B.-N. Guo, and C.-P. Chen, Some completely monotonic functions involving the gamma and polygamma functions, Journal of the Australian Mathematical Society 80 (2006), no. 1, 8188, RGMIA Research Report Collection 7 (2004), no. 1, article 5, 31-36, available online at http://rgmia.vu.edu.au/v7n1.html.

[10] J. G. van der Corput, Generalization of Carleman's inequality, Proceedings of the Section of Sciences, Koninklijke Akademie van Wetenschappen te Amsterdam 39 (1936), 906-911.

[11] H. van Haeringen, Completely monotonic and related functions, Report 93-108, Faculty of Technical Mathematics and Informatics, Delft University of Technology, Delft, 1993.

[12] B.-Ch. Yang, On a strengthened version of the more precise Hardy-Hilbert inequality, Acta Mathematica Sinica 42 (1999), no. 6, 1103-1110 (Chinese).

[13] On Hardy's inequality, Journal of Mathematical Analysis and Applications 234 (1999), no. $2,717-722$.

[14] On a relation between Carleman's inequality and Van der Corput's inequality, Taiwanese Journal of Mathematics 9 (2005), no. 1, 143-150.

[15] On an extension and a refinement of van der Corput's inequality, to appear in Chinese Quarterly Journal of Mathematics.

Jian Cao: School of Mathematics and Informatics, Henan Polytechnic University, Jiaozuo City, Henan Province 454010, China

E-mail addresses: 21caojian@163.com; goodfriendforeve@163.com

Da-Wei Niu: School of Mathematics and Informatics, Henan Polytechnic University, Jiaozuo City, Henan Province 454010, China

E-mail addresses: nnddww@163.com; nnddww@tom.com

Feng Qi: Research Institute of Mathematical Inequality Theory, Henan Polytechnic University, Jiaozuo City, Henan Province 454010, China

E-mail addresses: qifeng@hpu.edu.cn; fengqi618@member.ams.org; qifeng618@hotmail.com; qifeng618@msn.com; 316020821@qq.com 


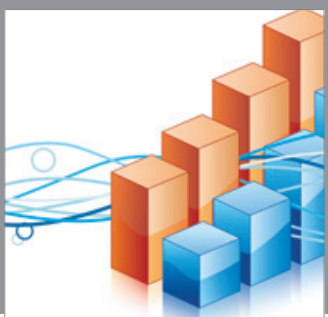

Advances in

Operations Research

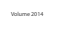

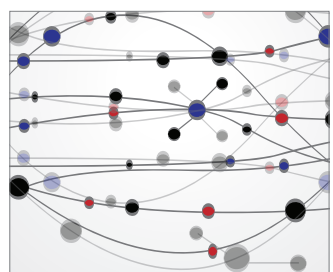

\section{The Scientific} World Journal
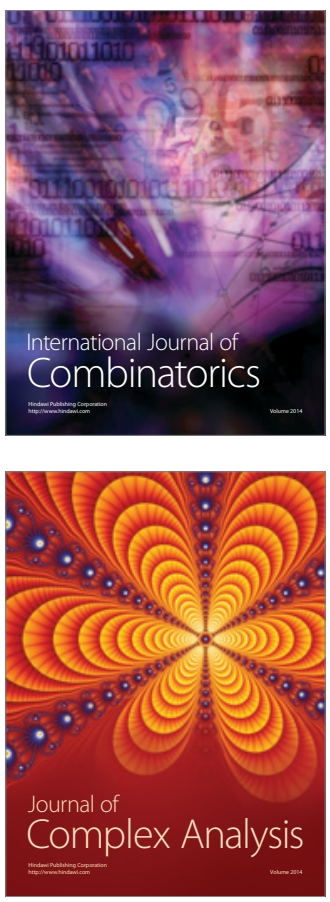

International Journal of

Mathematics and

Mathematical

Sciences
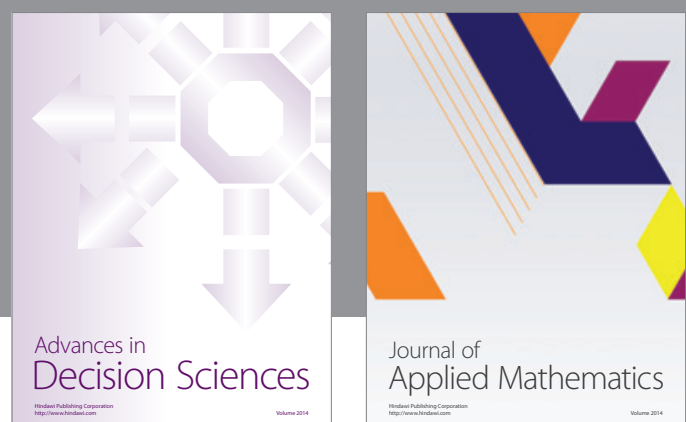

Journal of

Applied Mathematics
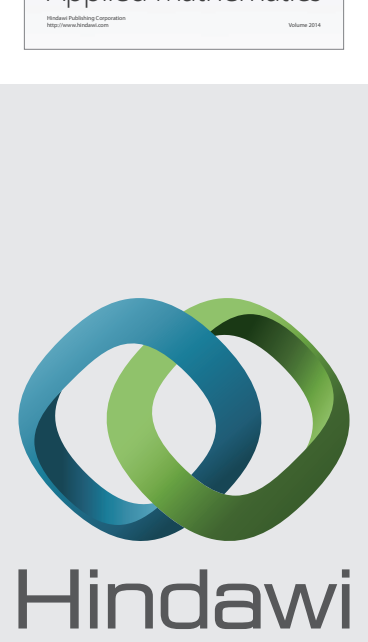

Submit your manuscripts at http://www.hindawi.com
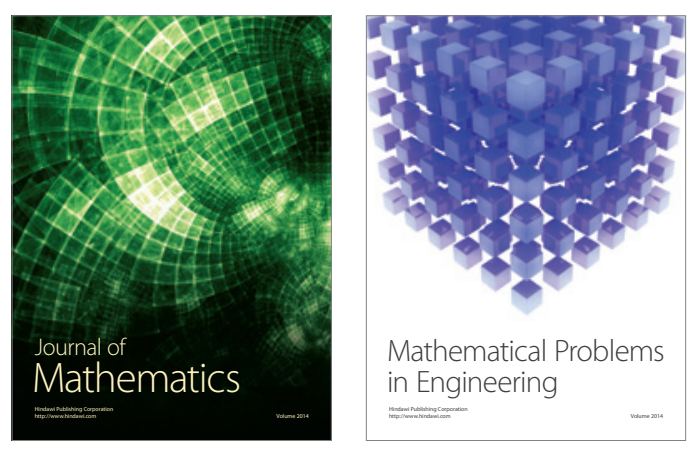

Mathematical Problems in Engineering
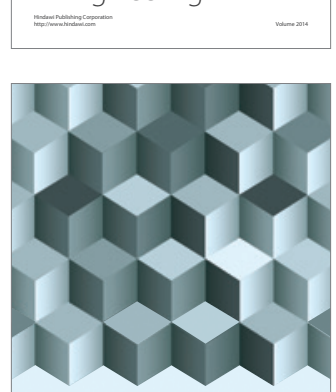

Journal of

Function Spaces
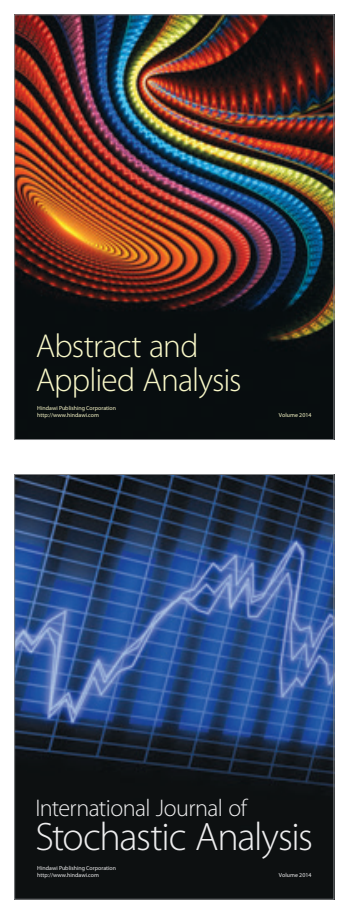

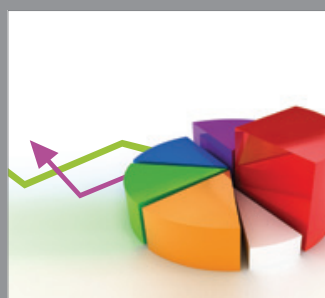

ournal of

Probability and Statistics

Promensencen
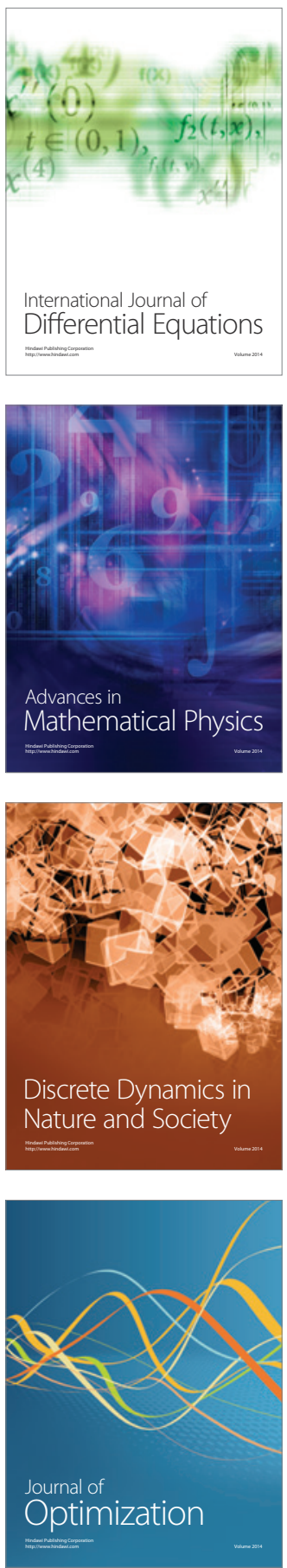